\title{
Rheological Properties of 1,4-Polyisoprene over a Large Molecular Weight Range
}

\section{Mahmoud Abdel-Goad, ${ }^{\dagger}$ Wim Pyckhout-Hintzen, ${ }^{*, \dagger}$ Stefan Kahle, ${ }^{\dagger} \mathrm{J}$ urgen Allgaier, ${ }^{\dagger}$ Dieter Richter, ${ }^{\dagger}$ and Lewis J . Fetters ${ }^{\ddagger}$}

Forschungzentrum J ülich, IFF, 52425 J ülich, Germany, and Department of Chemical and Biomolecular Engineering, Cornell University, I thaca, N ew York 14853-5021

Received November 10, 2003; Revised Manuscript Received February 5, 2004

\begin{abstract}
The dynamic viscoelastic properties of anionically polymerized model 1,4-polyisoprene (PI) with $6-8 \% 3,4$ microstructure and in the range of molecular weights between $1.5 \times 10^{3}$ and $3.2 \times 10^{6}$ $\mathrm{g} / \mathrm{mol}$ were reanalyzed using linear rheology. Viscosities obtained in the zero shear-rate limit, $\eta_{0}$, up to sufficiently high molecular weight PI samples indicated a clear transition to the pure reptation regime. All characteristic molecular weights, dividing the $\left[\eta_{0}, \mathrm{M}\right]$-interval in three different regimes could be obtained in high accuracy and a new "triangle" is proposed. The experimental data were analyzed in terms of the empirical Winter-relaxation BSW-model description for both dynamic moduli. The BSW parameters are determined and interpreted in terms of the packing model where possible. Limitations of the packing model are detected and discussed. Additionally, the parameters of the assumed distribution of relaxation times are compared to predictions of microscopic dynamic theories and useful correlations made. The publication deals, in part, with a misconception in the literature for estimates of the constraint release contribution. The monomeric friction coefficient $\xi_{0}$, determined in this study for the first time rheologically for anionic PI itself agrees with the natural rubber analogue.
\end{abstract}

\section{Introduction}

The relationship between rheological properties of polymers and their molecular structure has been a topic of intensive investigations in the past decades and has led to the development of sophisticated molecular theories as well as less-demanding though very useful approaches. Therefore, rheol ogical measurements have now become a common and basic analytical tool of polymer characterization. ${ }^{1-3}$ Microscopically, the viscoel asticity of polymers is apparently well described by the reptational tube model with corrections for timedependent topological fluctuations. Also, it is a wellknown fact that the melt flow behavior of polymers is very sensitive to their molecular weight and their molecular weight distribution. A typical example for this is the relation between the melt viscosity, $\eta_{0}$, and the molecular weight of linear polymers. Three different "characteristic" molecular weights cause a different behavior in the viscosity, given as $\eta_{0}=\mathrm{KM}^{\alpha}$. With increasing length of the chain, $\alpha$ varies from $\sim 1$ to $\sim 3.4$ to finally settle at 3 , respectively, when pure reptation sets in. This chain length dependence provides the link to the molecular structure and allows correlations between structure and properties to be detected. In this work we have reinvestigated the rheological properties of a series of anionically polymerized polyisoprene (PI) samples over a wide range of molecular weights (up to $3000 \mathrm{~K} \mathrm{~g} / \mathrm{mol}$ ) with the main attention directed toward higher molecular weights which have only scarcely (or not at all) been studied in detail. ${ }^{5}$

\section{Theoretical and Empirical Background}

Real entangled polymers exhibit a broad spectrum of relaxation times due to the high flexibility and associated freedom to many polymer chain configurations.

* Corresponding author. E-mail: w.pyckhout@fz-juelich.de. † Forschungzentrum J ülich, IFF.

‡ Cornell University.
Their shear relaxation modulus $\mathrm{G}(\mathrm{t})$ can be conveniently expressed by a weighed sum of corresponding terms for individual basic relaxation elements as

$$
G(t)=\sum_{i=1}^{n} G_{i} e^{-t / \tau_{i}}
$$

Here, $G_{i}$ and $\tau_{i}$ are the amplitude and characteristic times for each relaxing element. In this publication we have omitted on purpose the normal mode representation ${ }^{5}$ which is the molecular microscopic description of the relaxation time spectrum on a higher computational level. I nstead, an attempt is made to upgrade a former simple mechanical model and to compare this to modern understanding. The development of the above concept to include an infinite number of discrete elements leads to models in terms of continuous relaxation spectra $\mathrm{H}(\tau)$ and is applied to deliver useful semiquantitative information. The sum above in eq 1 is easily replaced by the integral. Under dynamic load the following relationships for real storage, $G^{\prime}$, and imaginary loss modulus, G", from Fourier transformation of $\mathrm{G}(\mathrm{t})$ then can be obtained as

$$
\begin{gathered}
\mathrm{G}^{\prime}(\omega)=\mathrm{G}_{\mathrm{e}}+\int_{-\infty}^{+\infty} \mathrm{H}(\tau) \omega^{2} \tau^{2} /\left(1+\omega^{2} \tau^{2}\right) \mathrm{d}(\ln \tau) \\
\mathrm{G}^{\prime \prime}(\omega)=\int_{-\infty}^{+\infty} \mathrm{H}(\tau) \omega \tau /\left(1+\omega^{2} \tau^{2}\right) \mathrm{d}(\ln \tau)
\end{gathered}
$$

from which the zero-shear rate viscosity follows in the low-frequency limit as

$$
\eta_{0}=\lim _{\omega \rightarrow 0}\left[\frac{\mathrm{G}^{\prime \prime}}{\omega}\right]=\int_{-\infty}^{+\infty} \mathrm{H}(\tau) \tau \mathrm{d}(\ln \tau)
$$

For un-cross-linked melts at infinite time $\tau=\infty$, the permanent elastic contribution $\mathrm{G}_{\mathrm{e}}$ is 0 . The terminal behavior for $\tau \rightarrow \infty$ and $\omega \rightarrow 0$ becomes clear from eqs 2 and 3 and shows that $\mathrm{G}^{\prime \prime}(\omega) \sim \omega$ and $\mathrm{G}^{\prime}(\omega) \sim \omega^{2}$. 
The $\mathrm{H}$-spectrum used was empirically expressed for the first time by Winter et al. 2,3 It is now widely used and known under the acronym of the BSW spectrum. For details, we refer the reader to the original literature. Different approaches to spectra are available, however. ${ }^{31}$ In short, the spectrum $\mathrm{H}(\tau)$ is given as

$$
\begin{array}{ll}
\mathrm{H}(\tau)=\mathrm{H}_{\mathrm{e}} \tau^{\mathrm{n}_{\mathrm{e}}}+\mathrm{H}_{\mathrm{g}} \tau^{-\mathrm{n}_{\mathrm{g}}} & \text { for } \tau_{0}<\tau<\tau_{\max } \\
\mathrm{H}(\tau)=0 & \text { for } \tau>\tau_{\max }
\end{array}
$$

$\mathrm{H}_{\mathrm{e}}$ represents the entangl ement regime and is defined by

$$
\mathrm{H}_{\mathrm{e}}=\mathrm{n}_{\mathrm{e}} \mathrm{G}_{\mathrm{N}}^{0} \tau_{\max }^{-\mathrm{n}_{\mathrm{e}}}
$$

The second term of $\mathrm{H}(\tau), \mathrm{H}_{\mathrm{g}}$ represents the glassy transition zone and in a similar way is written as

$$
\mathrm{H}_{\mathrm{g}}=\mathrm{n}_{\mathrm{g}} \mathrm{G}_{\mathrm{N}}^{\circ} \tau_{0}^{\mathrm{n}_{\mathrm{g}}}
$$

The spectrum is valid only above a shortest time $\tau>\tau_{0}$ of the experimental data and is set likewise equal to zero above the longest time $\tau_{\max }$. The elementary time $\tau_{0}$ compares to the longest Rouse time of an entanglement segment; i.e., it is linked to a chain of the size $\mathrm{M}$ $=\mathrm{M}_{\mathrm{e}}$ whereas $\tau_{\max } \sim \tau_{\mathrm{d}}$, on the other hand, represents the reptation time for the full chain, i.e., to have left and rebuilt its own configurational tube. The exact definition of the timescales is not strict due to the purely phenomenological basis of $\mathrm{H}$ nor is it strongly distinguished between $\tau_{\mathrm{e}}$ and $\tau_{\mathrm{c}}$ for $\tau_{0}$ in the onset of the 3.43.8 power for the viscosity $-\mathrm{M}_{\mathrm{w}}$ relationship. ${ }^{5}$ The number of parameters (five, of which two slopes seem to be fairly constant) is higher than the microscopic theory which makes it more flexible. For the purpose of this approach this is sufficient, however. Additionally, it can easily be shown that the same slopes, $n_{e}$ and $n_{g}$, which are prominent in the $\mathrm{H}$-spectrum also re-appear in the frequency dependence of the loss modulus G"$(\omega)$. Therefore, $n_{e}$ is the high- $\omega$ slope of $G^{\prime \prime}(\omega)$ in the entanglement regime, $n_{g}$ is the empirical slope of $\mathrm{G}^{\prime}(\omega)$ as well as $\mathrm{G}^{\prime \prime}(\omega)$ in the dynamic glass transition regime. $\mathrm{n}_{\mathrm{g}}=1 / 2$ is expected theoretically from the Rouse theory at short times $\left(\omega^{+1 / 2}\right)$ whereas $n_{e}=1 / 2$ is calculated from the pure reptation model $\left(\omega^{-1 / 2}\right)$. The appearance of seemingly smaller slopes in the crossover region is partly due to the mixing of both regimes. In the case of a single relaxing Maxwell element both times $\tau_{\max }$ and $\tau_{\mathrm{d}}$ are identical whereas for a distribution of times, we expect $\tau_{\max }>\tau_{\mathrm{d}}$. The easy-determinable slopes allow the necessary link to molecular theories to be made. $\mathrm{G}_{N}^{0}$ in eqs 6 and 7 is the plateau modulus and contains the length scale of the reptation tube confinement. It is typically obtained from the plateau of $\mathrm{G}^{\prime}(\omega)$ for $\omega \rightarrow \infty$ (neglecting the high-frequency part of the spectrum) and is defined in analogy to rubber elasticity as:6,7

$$
G_{N}^{o}=\frac{\rho R T}{M_{e}}=\frac{\left\langle R^{2}\right\rangle_{0} \rho R T}{M d_{t}^{2}}=\frac{k T}{V_{e}}
$$

where $\rho, M, d_{t}$, and $V_{e}$ are respectively density, chain molecular weight, the diameter of the tube $(\sim 21$ times packing length), and the entanglement volume $\left(\mathrm{M}_{\mathrm{e}} \rho \mathrm{N}_{\mathrm{a}}\right)$. Here, the mean squared end-to-end distance, related to a mass $M$, is given as $\left\langle R^{2}\right\rangle_{0}$. We stick to the original convention and discard the suggested $4 / 5$ prefactor. $4,8,9$
Due to the obvious connection of modulus with microscopic and structural details of the polymer it appeared worthwhile to further evaluate the applicability of the packing model as initiated by Fetters. ${ }^{6,7}$ In particular, the high $\mathrm{M}_{\mathrm{w}}$ side remained unexplored in terms of the packing parameter.

The packing concept as introduced ${ }^{6,7}$ in the past decade combines chain entangling properties with coiling properties and introduces the packing length $p$ as

$$
p=\frac{M}{\left\langle R^{2}\right\rangle N_{a} \rho}=\pi \frac{h^{2}}{4 I_{k}}(\AA)
$$

The chain dimensions are often expressed in terms of $\mathrm{C}_{\infty}$ (Flory's characteristic ratio), which is a reflection of chain configurational behavior. This expression relates $p$ to polymer density and the unperturbed chain dimension and in turn to the effective chain diameter, $h$, and the Kuhn step length, $I_{k}\left(I_{k}=I_{0} C_{\infty}\right.$ where $I_{0}$ is the length of the backbone bond. The polymer chain may then be considered to consist of cylindrical units with a diameter of $h$ and $a$ length of $I_{k}$. The cylinder diameter expressed in terms of $\mathrm{h}^{10,11}$ is

$$
\mathrm{h}=2\left[\mathrm{~m}_{\mathrm{b}} / \pi \rho \mathrm{N}_{\mathrm{a}} \mathrm{l}_{0}\right]^{0.5}\left(\AA^{2}\right)
$$

where $m_{b}$ is the average mol ecular weight per backbone bond.

The packing model has been extensively used and evaluated. This work was thus initiated to evaluate further predictions. The packing length for 1,4-PI for the particular microstructure has been taken for $25{ }^{\circ} \mathrm{C}$ from the literature as $p=3.10 \AA$ for the $6-8 \% 3,4$ microstructure while $\rho=0.90 \mathrm{~g} \mathrm{~cm}^{-3}$. The following empirical expressions ${ }^{6,7,12}$ using eq 8 have been developed relating the packing length to the characteristic polymer molecular weights:

$$
\begin{aligned}
M_{e}= & 261 \rho N_{a} p^{3} \exp (T / 635) \\
M_{c} & =M_{e}\left[\frac{p^{*}}{p}\right]^{0.65} \\
M_{r} & \cong M_{e}\left[\frac{p^{*}}{p}\right]^{3.9}
\end{aligned}
$$

where $p^{*}=9.2 \AA$ and $T$ in absolute $K .{ }^{7}$ Equation $11 \mathrm{c}$ is based on some assumptions which will be reevaluated here (strictly speaking, the packing length has been linked in a rigorous fashion only to $\mathrm{Me}_{\mathrm{e}}$ ). At $\mathrm{p}^{*}$ the entanglement and critical molecular weights become equal. The $M_{e}$ expression is linked to a wide series of different polymers and partly to assumptions of dynamic processes in the long time limit (as we will show later). The uncertainty on the exponents 0.65 and 3.9 is about 0.1 . The simplicity of these formulations makes the packing model very attractive and allows for accurate to semiquantitative predictions of various polymer properties.

By using the BSW spectrum and neglecting the high frequency part (due to Rouse-like relaxations in the glass transition) simple math leads additionally to a simple estimate for the zero-shear viscosity, $\eta_{0}$, as

$$
\eta_{0}=\int_{0}^{\tau_{\max }} \tau \mathrm{H}(\tau) \mathrm{d}(\operatorname{In} \tau)=\frac{\mathrm{n}_{\mathrm{e}}}{\mathrm{n}_{\mathrm{e}}+1} \mathrm{G}_{\mathrm{N}}^{0} \tau_{\max }
$$


Table 1. Molecular Characteristic of PI Samples by SEC and LALLS

\begin{tabular}{|c|c|c|c|}
\hline polymer & $10^{-3} \mathrm{M}_{\mathrm{w}}(\mathrm{GPC})$ & $M_{w} / M_{n}$ & $10^{-3} \mathrm{M}_{\mathrm{w}}(\mathrm{LS})$ \\
\hline PI1.5K & 1.5 & 1.09 & \\
\hline PI3K & 3.84 & 1.05 & \\
\hline PI7K & 7.38 & 1.04 & \\
\hline PI13K & 13.5 & 1.02 & \\
\hline PI22K & 22.5 & 1.02 & \\
\hline PI57K & 57.4 & 1.03 & \\
\hline PI80Ka & 75 & 1.06 & \\
\hline PI100Ka & 102 & 1.04 & 103 \\
\hline PI163Ka & 110 & 1.02 & \\
\hline PI 140Ka & 128 & 1.08 & 130 \\
\hline PI 190Ka & 198 & 1.03 & 202 \\
\hline PI300K & 293 & 1.02 & \\
\hline PI463K & 464 & 1.05 & $426^{b}$ \\
\hline PI570K & 576 & 1.09 & 570 \\
\hline PI730K & 735 & 1.04 & 751 \\
\hline PI950K & 963 & 1.12 & \\
\hline PI1000Ka & 1013 & 1.05 & \\
\hline PI3000K & 3000 & 1.23 & 3250 \\
\hline $\mathrm{PI} 3200 \mathrm{~K}^{\mathrm{a}}$ & 2910 & 1.15 & $3140^{c}$ \\
\hline
\end{tabular}

a Samples prepared in J ülich. Remainder were from PSS. b $5 \%$ of coupled product obtained via SEC. ' Derived from an internal intrinsic viscosity relationship and universal calibration for polydiene standards in THF at $25^{\circ} \mathrm{C}$. The accuracy of the value is not better than $10 \%$, but the molecular weight is corroborated by the dependence of relaxation times on $\mathrm{M}$ (see rheological section).

i.e. the melt viscosity is linearly related to the longest relaxation time, the prefactor being a function of slopes in the intermediate time-domain. ${ }^{13}$ An internal consistency for the plateau modulus is obtained from the slope of latter equation if $\eta_{0}$ is plotted vs $\tau_{\text {max }}$.

\section{Experimental Section}

Materials. All samples in this study were prepared by anionic polymerization in $\mathrm{FZ}$ J J ülich or were commercially obtained from Polymer Standards Lab (PSS), Mainz, Germany. Purified benzene was the polymerization solvent while purified sec-butyllithium was the initiator and degassed methanol was used for headgroup deactivation. The isoprene monomer was purified by stirring at room temperature over dibutylmagnesium followed by n-butyllithium at low temperature to remove possible remaining impurities. After termination, the polymers were precipitated in methanol and dried. BHT or HP136 as antioxidant was added to the methanol as to obtain a $0.1 \%$ level in the dry polymer. As a result, all samples could be synthesized with small polydispersity in the range of the expected polydispersity for anionically polymerized polymers as shown in Table 1. All samples were prepared in PTFE molds and shaped under vacuum at room temperature and slight pressure before mounting on the rheometer.

Material Characterization. The molecular weight and molecular weight distribution (Table 1) of the samples were determined via a $\mathrm{M}_{\mathrm{w}}$ calibration to static LALLS from standards using a Waters 150C SEC, equipped with differential refractometer, viscosity and light scattering detector operated at $25^{\circ} \mathrm{C}$ with tetrahydrofuran as the mobile phase. Depending on the time of preparation, the molecular weights were measured with respect to PS or PI as calibration standards and analyzed using the universal calibration technique. Crosschecks of $\mathrm{M}_{\mathrm{w}}$-determinations between J ülich and PSS samples revealed average deviations not larger than $5 \%$. The elution volumes of both series fitted to a general diene-calibration curve from PB and PI standards. In addition, static small angle laser light scattering (LALLS) from dilute solutions in cyclohexane or heptane at room temperature was used in this study to determine (selected) weight-average molecular weights in the Zimm or Berry approximation. In the case of sample PI $3200 \mathrm{~K}$, the Berry analysis result proved to be more appropriate to the linear regression. (Table 1). The molecular weight-SEC elution behavior of the polyisoprenes used in this work is given in Figure 1.

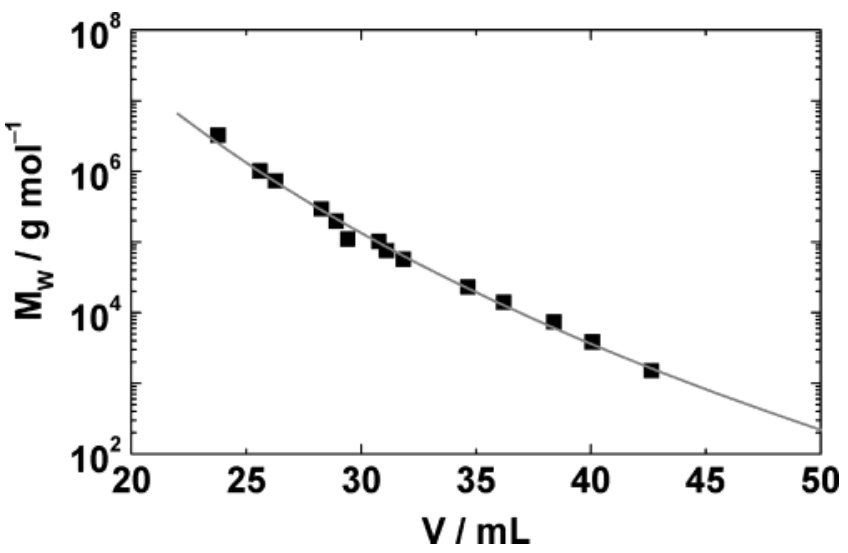

Figure 1. Elution values determined from the peak maximum for polyisoprene samples from low to high mol ecular weights. The solid line is only a guide to the eye. The experimental points follow closely the experimental polydiene calibration curve from SEC. The 100K sample is too low and would indicate that the $\mathrm{M}_{\mathrm{w}}$ is off by a factor of 2 .

Selected polymers were additionally characterized by $\mathrm{H}$ NMR measurements in deuteriochloroform. The spectra were recorded on a Bruker AMX 300 spectrometer. The content of the 1,4 and 3,4 structures was calculated from the signals arising from the ol efinic protons between 4 and 6 ppm and yiel ded a range between 5.5 and $7 \%$ or an average of $(6.3 \pm$ $1.2) \%$ on 9 samples with a large spread in $M_{w}$. The microstructure of these polyisoprenes can be assumed to be virtually identical to those used by Gotro and Graessley. ${ }^{5}$

Rheological Characterization. Dynamic mechanical spectroscopy was used to determine the viscoelastic properties of the monodisperse $\mathrm{PI}$ samples over a wide range of the molecular weights from about $1 \mathrm{~K}$ up to $3000 \mathrm{~K} \mathrm{~g} / \mathrm{mol}$. The rheological measurements were performed on a straincontrolled Rheometric Sci. ARES system operated in the dynamic mode with 8 and $25 \mathrm{~mm}$ parallel plates and a 2KFRTN 1 force rebalance transducer. The smaller tools were used for the highest $\mathrm{M}_{\mathrm{w}}$ sample. Cone-plate geometry was used for the $22 \mathrm{~K}$ sample and lower as the corrections for parallel plates were not negligible. For $13 \mathrm{~K}$ no frequency dependence of the viscosity could be spotted. Geometry effects were not present from tests on intermediate samples. The loading temperature was generally $25^{\circ} \mathrm{C}\left(50^{\circ} \mathrm{C}\right.$ for high $\left.\mathrm{M}_{w}\right)$ and pre-shaped samples relaxed to exceed the estimated terminal time by about 2 orders of magnitude. The high $M_{w}$ sample was relaxed overnight to vanishing normal force. All samples were tested typically over a temperature range of -50 to $+140{ }^{\circ} \mathrm{C}$ under a nitrogen blanket. For molecular weights above 750K the full accessible frequency range of 0.001-100 $\mathrm{rad} / \mathrm{s}$ was used whereas the lower $0.01 \mathrm{rad} / \mathrm{s}$ was taken for the low to intermediate molecular weights up to $600 \mathrm{~K}$. This reduces determination of melt viscosities from shifted data. Temperature stability was about $0.5^{\circ} \mathrm{C}$. Thicknesses were about $1 \mathrm{~mm}$ initially and varied with temperature as to keep the normal force measurement constant. The strain amplitude was chosen $1 \%$ to ensure linear response over the whole $M_{w}$ range and was increased only to $5-20 \%$ for the low $M_{w}$ materials below 3K.

Special care was taken to compare all samples at a constant $\left(T_{0}-T_{g}\right)$ difference above the glass temperature, $T_{g}$, for the different molecular weight samples. Here, $T_{0}$ is the reference temperature. Doing this, in a good approximation the monomeric friction coefficient becomes constant and automatically free chain ends are corrected for. Therefore, an empirical equation was fitted in this study using experimental $T_{g}$ values reported in the literature. ${ }^{5,14-17}$ This equation relates $T_{g}$ to $M_{n}$ ( $\mathrm{T}_{\mathrm{g}}$ in absolute temperature) as $1 / \mathrm{T}_{\mathrm{g}}=0.00484+0.42959 / \mathrm{M}_{\mathrm{n}}$. The use of $M_{n}$ over $M_{w}$ is arbitrary due to the small polydispersity index. The respective $\mathrm{T}_{\mathrm{g}}$ for all of the $\mathrm{PI}$ samples was calculated from this equation. $T_{g}$ is situated between -66 and 


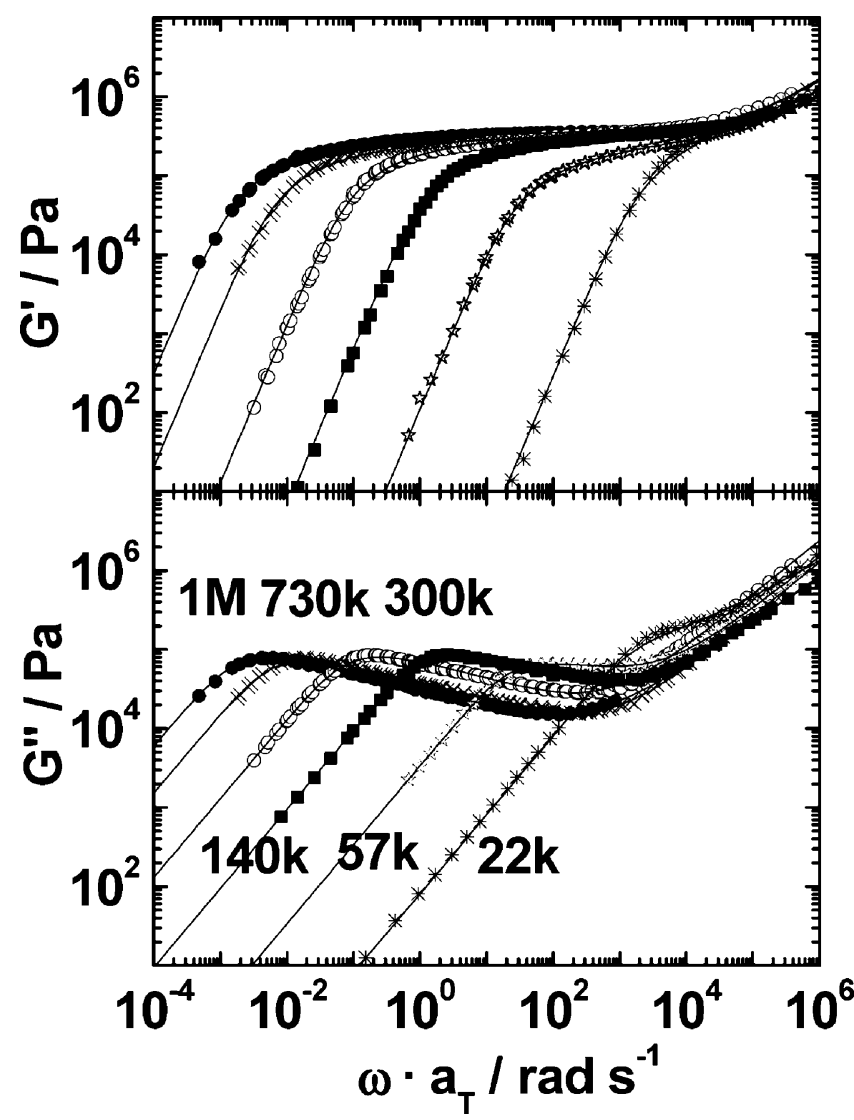

Figure 2. Selected master curve of $\mathrm{G}^{\prime}$ and $\mathrm{G}^{\prime \prime}$ for $\mathrm{PI} 22 \mathrm{~K}$, PI57K, PI140K, PI300K, PI730K, and PI1000K from right to left. The solid line through the experimental data represents the best BSW fit as discussed in the text. Fits were mainly obtained on G" and those corresponding to theoretical functions for $\mathrm{G}^{\prime}$ (Kramers-Kronig) were calculated.

$-78^{\circ} \mathrm{C}$. $\mathrm{T}_{0}-\mathrm{T}_{\mathrm{g}}$ was chosen to be $91^{\circ} \mathrm{C}$. The dependence of $\mathrm{T}_{\mathrm{g}}$ on $M_{n}$ vanishes for $M_{n} \sim 10^{4} \mathrm{~g} / \mathrm{mol}$, and the validity of the relationship was tested for some samples below this limit.

The master curves of both dynamic moduli of each sample to their respective $T_{0}$ were constructed by means of the timetemperature superposition principle using the relationship described by Williams-Landel-Ferry ${ }^{18}$ as

$$
\log a_{T}=\log \frac{\tau(T)}{\tau\left(T_{0}\right)}=\frac{-C_{1}\left(T-T_{0}\right)}{C_{2}+\left(T-T_{0}\right)}
$$

$a_{T}$ is the experimentally derived shift factor, obtained from 2-dimensional shifting of all curves using the Rheometric Scientific software. The average WLF-constants $C_{1}$ and $C_{2}$ varied between 4.2 and 4.8, respectively. 130 and 142 to give averages $4.5 \pm 0.3$ and $136 \pm 6$. The higher values were found for the lowest molecular weights. The reference temperature was nominally $25^{\circ} \mathrm{C}$ but fluctuations of $0.5^{\circ} \mathrm{C}$ here were noted. Taking the extrema for both parameters, however, the maximum shift factor ratio deviation is about $40 \%$, i.e., a factor of 1.4. This shift does not apply to the medium up to low frequency range as this is fully covered by the reference temperature but only affects the dynamical glass transition/ Rouse branch. Indeed, a close inspection of Figure 2 reveals therefore some scatter at high frequencies comparable to $\omega \tau_{\mathrm{e}}$ $=1$ which may be due to accumulated errors by (1) the use of separate shift factors per sample and (2) temperature fluctuations and $\mathrm{T}_{\mathrm{g}}$ corrections and (3) possible intrinsic dynamic instabilities of the transducer and last but not least (4) physical reasons like nonsimple thermorheological behavior. If additionally a straight line is fitted through $\mathrm{G}^{\prime \prime}(\omega)$ which intersects the modulus at $10^{5} \mathrm{rad} / \mathrm{s}$, the intersection varies consistently with $\mathrm{M}_{\mathrm{w}}$ except for the $300 \mathrm{~K}$ sample which behaves differently. The same slope at the intersection is obtained for theoretical expectations of the model for the same entanglement degree. Even if the experimental scatter by WLF shifting by factors from the flow region is larger than one would wish, the final results are not affected. First of all, zeroshear viscosities are almost all directly contained in the roomtemperature measurement (and therefore independent of the shifting quality) and second, the proposed phenomenological model in this work contains a basic microscopic step time $\tau_{\mathrm{e}}$ which determines the uprise of the modulus, here as an adjustable parameter-unlike in ref 19-and no constraint with the longest relaxation time of the polymer had to be assumed. $\mathrm{T}_{0}$ was chosen to be $25^{\circ} \mathrm{C}$ for this set of experiments (unless stated otherwise). The vertical shift factors b- were typically in the range 0.8 to 1.1 as expected from density and temperature factors. They are approximately following $\mathrm{b}_{\top}=8.21$ $\exp (-0.0089 T)+0.46$ (T absolute)

\section{Results and Discussion}

In Figure 2 the storage modulus, $\mathrm{G}^{\prime}(\omega)$, as well as master curves of $\mathrm{G}^{\prime \prime}(\omega)$ are logarithmically plotted against the angular frequency $\omega$ for selected samples. The G" curve shows the terminal peak at the lowfrequency branch. In the terminal zone, the curves show slopes of $1.0\left(G^{\prime \prime} \sim \omega\right)$ as expected for simple flow and monodisperse polymers that lack long-rel axing components like gels or branched structures. Only the sample PI463K (not shown) showed a contribution of the coupling impurity upon termination of the anionic polymerization which could be safely excluded, however, from the fits due to the shift of about 1 decade (i.e., $2^{3}$ ) to lower frequencies and contributing with an estimated amplitude to $G^{\prime \prime}$ with only $\phi^{2}$ (i.e., 0.052). $\phi$ is the volume fraction of the double-length polymer. The low molecular weight samples had either small fractions of impurities or ran into resolution problems as is clearly visible. The terminal zone, however, could not be accessed for some high molecular weight samples as in the cases of PI3000K and PI3200K (not shown) due to technical limitations. It sufficed for this work, however, to determine the peak position in the loss modulus to estimate the melt viscosity and use the linear relationship with $\tau_{\mathrm{d}}$ or $\tau_{\text {max }}$.

The experimental data were analyzed by fitting nonlinearly the BSW spectrum for $\mathrm{G}^{\prime \prime}(\omega)$ and/or $\mathrm{G}^{\prime}(\omega)$ as shown in Figure 2 for some representative examples. Excellent fits over the fully explored molecular weight range with five consistent model parameters could be achieved and are summarized as $\mathrm{G}_{\mathrm{N}}^{\circ}=0.372 \pm 0.006$ $\mathrm{MPa}, \tau_{0}=(1.64 \pm 0.5) \times 10^{-5} \mathrm{~s}, \mathrm{n}_{\mathrm{g}}=0.66 \pm 0.04$, and $\mathrm{n}_{\mathrm{e}}=0.23 \pm 0.02$. The full BSW parameter sets for all available samples are listed in Table 2 . The standard deviations from fitting the longest relaxation times $\tau_{\max }$ to the data varied between $2 \%$ (for high M) to about 20\% in the range $\mathrm{M} \sim \mathrm{M}_{\mathrm{c}}$. F or the lowest molecular weights, however, the melt viscosity was obtained directly from theterminal Newtonian region without fitting of theloss modulus in the terminal regime. All BSW parameters for the low molecular weight samples are taken to be of lower accuracy except for the melt viscosity $\eta_{0}$ since the Newtonian plateau in $\eta\left(\eta_{0}\right)$ at very low-frequency i.e., low shear rate is highly pronounced for these samples. For all others the zero-shear rate viscosity values in the table are obtained from the fitted $G^{\prime \prime}(\omega)$ curves and the theoretical limiting plateau for $\omega \rightarrow 0$ in the representation of eq 4. The quoted viscosity error bars are determined by the fit quality of the full frequency range with the underlying spectrum. Theoretical val ues $\eta_{0, \text { theo }}$ listed in the table are obtained from the relationship eq 12 using $n_{e}$ and serve well as an independent measure for the relaxation time spectrum by Winter. 
Table 2. BSW Parameters from $\mathbf{G}^{*}(\omega)$ of PI at $\mathbf{T}=25^{\circ} \mathbf{C}$

\begin{tabular}{|c|c|c|c|c|c|c|c|c|c|c|c|c|}
\hline polymer & $\begin{array}{c}\mathrm{G}_{\mathrm{N}}^{\circ} \\
(\mathrm{MPa})\end{array}$ & $\begin{array}{c}\tau_{\max } \\
(\mathrm{s})\end{array}$ & $\mathrm{n}_{\mathrm{g}}$ & $\mathrm{n}_{\mathrm{e}}$ & $\begin{array}{c}\eta_{0} \\
\text { (Pa s) }\end{array}$ & $\begin{array}{l}\tau_{d}^{\infty} \\
(\mathrm{s})\end{array}$ & $\begin{array}{l}\eta_{0, \text { theo }} \\
\text { (Pa s) }\end{array}$ & $\begin{array}{c}\tau_{\mathrm{d}}\left(\omega_{\max }\right) \\
(\mathrm{s})\end{array}$ & Z & $10^{-3} \mathrm{M}_{\mathrm{w}}$ & $\mathrm{C}_{1}$ & $\mathrm{C}_{2}(\mathrm{~K})$ \\
\hline PI3200K & & & & & $\left(2.15 \times 10^{9}\right)$ & & $2.15 \times 10^{9}$ & 6135 & 500 & 3140 & (3.4) & (107) \\
\hline PI3000K & 0.5 & $1.80 \times 10^{4}$ & 0.56 & 0.11 & $\left(1.71 \times 10^{9}\right)$ & 8240 & $1.71 \times 10^{9}$ & 3600 & 500 & 3250 & (3.4) & (107) \\
\hline PI1000K & 0.37 & $4.79 \times 10^{2}$ & 0.68 & 0.24 & $3.39 \times 10^{7}$ & 243 & $3.56 \times 10^{7}$ & 237 & 169 & 1013 & 3.73 & 131.0 \\
\hline PI930K & 0.37 & $6.60 \times 10^{2}$ & 0.63 & 0.22 & $3.39 \times 10^{7}$ & 338 & $4.91 \times 10^{7}$ & 237 & 169 & 963 & 3.71 & 130.5 \\
\hline PI730K & 0.35 & $2.29 \times 10^{2}$ & 0.68 & 0.21 & $1.44 \times 10^{7}$ & 122 & $1.70 \times 10^{7}$ & 74 & 122 & 735 & 3.73 & 108.6 \\
\hline PI570K & 0.35 & 99.5 & 0.60 & 0.23 & $6.50 \times 10^{6}$ & 56.3 & $6.51 \times 10^{6}$ & 25.7 & 90 & 576 & 4.15 & 114.4 \\
\hline PI463K & 0.38 & 72.6 & 0.62 & 0.23 & $5.05 \times 10^{6}$ & 42.7 & $5.16 \times 10^{6}$ & 17.5 & 74 & 464 & 4.64 & 132.7 \\
\hline PI300K & 0.38 & $1.80 \times 10^{1}$ & 0.72 & 0.23 & $1.20 \times 10^{6}$ & 11.6 & $1.34 \times 10^{6}$ & 6.06 & 49 & 293 & 4.39 & 131.29 \\
\hline PI190K & 0.39 & $4.90 \times 10^{0}$ & 0.66 & 0.19 & $3.18 \times 10^{5}$ & 3.54 & $3.64 \times 10^{5}$ & 1.08 & 33 & 198 & 4.83 & 138.2 \\
\hline PI140K & 0.38 & $0.119 \times 10^{1}$ & 0.58 & 0.26 & $9.36 \times 10^{4}$ & 1.03 & $8.85 \times 10^{4}$ & 0.32 & 21 & 128 & 4.67 & 133.8 \\
\hline PI163K & 0.32 & 0.7 & 0.70 & 0.17 & $3.77 \times 10^{4}$ & 1.60 & $5.94 \times 10^{4}$ & 0.17 & 18 & 110 & 4.63 & 133.8 \\
\hline PII00K & 0.42 & $2.4 \times 10^{-1}$ & 0.60 & 0.19 & $1.65 \times 10^{4}$ & 0.60 & $1.78 \times 10^{4}$ & 0.05 & 17 & 102 & 4.63 & 133.6 \\
\hline PI80K 2 & 0.43 & $1.19 \times 10^{-1}$ & 0.64 & 0.22 & $1.04 \times 10^{4}$ & 0.144 & $8.85 \times 10^{3}$ & 0.05 & 12 & 75 & 5.00 & 141.8 \\
\hline PI57K & 0.33 & $6.00 \times 10^{-2}$ & 0.65 & 0.19 & $3.37 \times 10^{3}$ & & $4.46 \times 10^{3}$ & & & 57 & 5.00 & 140.3 \\
\hline $\mathrm{PI} 22 \mathrm{~K}$ & 0.30 & $0.8 \times 10^{-3}$ & 0.48 & 0.21 & $8.99 \times 10^{1}$ & & 149 & & & 23 & 5.57 & 151.2 \\
\hline PI13K & 0.32 & $2.0 \times 10^{-3}$ & 0.36 & 0.086 & $1.04 \times 10^{1}$ & & 149 & & & 14 & & \\
\hline PI7K & & & & & 6.497 & & & & & 7.38 & & \\
\hline PI4K & & & & & 2.197 & & & & & 3.84 & & \\
\hline PIIK & & & & & 1.180 & & & & & 1.50 & & \\
\hline
\end{tabular}

The plateau modulus, $\mathrm{G}_{N}^{\circ}$, on the contrary, is a quantity which is more interesting in view of the relationships to the packing model and the tube model approach. The Kramers-Kronig relation of the loss modulus to give $G_{N}^{\circ}$ cannot be applied here due to considerable interfering and mixing contribution of the Rouse-transition regime and therefore extensive use will be made of the assumed power law relaxation spectrum for its determination. The plateau modulus enters naturally in both dynamic moduli and can be determined from both with different accuracy. It was advantageous to do on the basis of G" primarily since at the same time the fitted exponents $n_{e}$ and $n_{g}$ have their visible counterpart in the $\omega$-domain. Using the parameters of the more sensitive G" the storage modulus can be calculated accordingly.

From Table $2, \mathrm{G}_{\mathrm{N}}^{\circ}=0.372 \pm 0.006 \mathrm{MPa}$ is in good agreement with literature values. ${ }^{5-7}$ The values for the frequency dependence of relaxation time exponents $n_{e}$ and $\mathrm{n}_{\mathrm{g}}$ are statistically distributed over all samples and yield on average $n_{e}=0.23 \pm 0.02$ and $n_{g}=0.66 \pm 0.04$. They were claimed to be universal exponents in the past but nowadays merely are the indicators for basic dynamic mechanisms. Pure reptation as in the original Doi-Edwards ${ }^{8,9}$ work would, e.g., predict $n_{e}=1 / 2$, that means $\omega^{-1 / 2}$ in the high-frequency slope of the loss peak. This changes over to $n_{e}=1 / 4$ if contour length fluctuations additionally affect the reptation time. This means that the peak position in the loss modulus moves to higher frequencies. ${ }^{14,15}$ This study reveals the latter to be pronounced in the relaxation of linear chains. Here, we believe that it is contour length fluctuations (CLF) that effectively reduce the length of the tube and therefore speed up the reptation process. $4,19,20$ It is a well-known and accepted reason for the unexpected dependence of viscosity on $\mathrm{M}$ and the approximate 3.4 (or similar) exponent. Note that the slope yields only an effective exponent which varies from publication to publication and polymer to polymer and still lacks any deep interpretation. Recently, constraint-rel ease (CR) effects, due to the motion of the configurational tube itself al so were shown to vary with the time scale of the reptation time. ${ }^{20}$ These processes are connected to displacements of the tube when a topological constraint is removed through reptation. A simple, easy-applicable and quantitative treatment of CR is not readily available yet and is thus neglected at this point. It will be a competing mechanism to consider ${ }^{21}$ if the contour length fluctuation mechanism has become ineffective. Depending on the degree of sophistication, the former CLF mechanism functions to reduce the reptation time from $\tau_{\mathrm{d}}{ }^{\infty}$ to $\tau_{\mathrm{d}}$ as ${ }^{4,19,20}$

$$
\tau_{\mathrm{d}}=\tau_{\mathrm{d}}^{\infty}\left(1-\frac{\mathrm{C}}{\sqrt{\mathrm{Z}}}\right)^{2} \approx \tau_{\mathrm{d}}^{\infty}\left(1-\frac{2 \mathrm{C}}{\sqrt{\mathrm{Z}}}+\frac{\mathrm{C}^{2}}{\mathrm{Z}}\right)
$$

Here, the numerical constant $C$ is between 1 and 1.47 while the number of entanglements is defined as $Z=$ $\mathrm{M} / \mathrm{M}_{\mathrm{e}}$. For infinite $\mathrm{Z}$, the correction becomes 1 and therefore $\tau_{\mathrm{d}}{ }^{\infty}=\tau_{\mathrm{d}}$. Following the reptation concept, $\tau_{\mathrm{d}^{\infty}}$ $=3 Z^{3} \tau_{\mathrm{e}}$ in terms of the basic segmental time. The best approximation to eq 14 has recently been presented by Likhtman and McLeish ${ }^{19}$ where the coefficients are obtained reliably from simulation data. We note that this Likhtman model describes the dynamic moduli in terms of only three parameters. The use of only $\tau_{\mathrm{e}}, \tau_{\mathrm{d}}$, plateau modulus, and a few estimated CR-related constants is very constraining and demanding, however, and requires ideal data over the full interval. Somewhat larger C's are predicted from it. The tabulated longest relaxation times $\tau_{\max }$ can be rescaled appropriately in order to test the scaling of Rouse and reptation times. We note that the experimental longest time values have taken into account the mentioned mechanism indirectly being a fit parameter. At the same time the derived $n_{e^{-}}$ exponent $\sim 1 / 4$ confirms the contribution of CLF (and also $\mathrm{CR}$ ) to the stress relaxation. A simulation-based $\tau_{\mathrm{d}}{ }^{\infty}$ therefore can be obtained from the fit parameters using eq 14. From Table 2 the scaling $\tau_{\mathrm{d}}^{\infty} \sim Z^{3}$ is approximately retrieved for the different samples and yields on average $\tau_{\mathrm{e}}=(1.9 \pm 0.8) \times 10^{-5} \mathrm{~s}$. Well above $\omega \sim$ $\tau_{\mathrm{e}}^{-1}$, the slope of all moduli of the investigated samples as well as their amplitude will tend to the same dependence (see Experimental Section). The rheological $\tau_{\mathrm{e}}$ as well as $\tau_{0}$ in this study compares very well with the directly observed value for linear 1,4-polyisoprene determined from neutron spin-echo (NSE) measurements at $200{ }^{\circ} \mathrm{C} .22$ The NSE value is equival ent to $\tau_{\mathrm{e}}=$ $1.1 \times 10^{-5} \mathrm{~s}$ if reduced to $25^{\circ} \mathrm{C}$ using our averaged WLF shift factors since in the cited reference proper $C_{1}$ and $\mathrm{C}_{2}$ are missing. We recall that the minimum and maximal characteristic times, appearing in eq 6 and 7 and which determine the $\mathrm{H}$-spectrum along with the exponents, were optimized quantities. Their specific values are intimately connected with the numerical evaluation of the integrals and the assumption of the 
spectrum outside these limits. Since the melt viscosity is obtained analytically as a linear function of $n_{e}, G_{N}^{0}$, and $\tau_{\max }$ as shown in eq 12, we propose to generalize the result to allow the identification of spectrum variables with molecular rheology variables: For a single Maxwell element with a single relaxation time and $\tau_{\mathrm{d}}$ $=\tau_{\max }$ necessarily, the high $\omega$-wing of the terminal loss peak is found to vary with $\omega^{-1}$. This corresponds to $n_{e}$ $=1$ as can be deducted from eq 3 . The lower $\omega$-region still is $\omega^{1}$ which determines the zero-shear viscosity $\eta_{\text {o. }}$. Inserting $\mathrm{n}_{\mathrm{e}}=1$ in eq 12 yields $\eta_{\mathrm{o}}=1 / 2 \mathrm{G}_{\mathrm{N}}^{0} \tau_{\max }=$ $1 / 2 \mathrm{G}_{\mathrm{N}}^{0} \tau_{\mathrm{d}}$. If a full spectrum is taken into account, i.e., if the $n_{e}$ exponent is empirically correcting for reptation, CLF and CR contributions, the BSW approach yields with $\mathrm{n}_{\mathrm{e}}=1 / 4$ the simple relation $\eta_{\mathrm{O}}=1 / \mathrm{G}_{\mathrm{N}}^{\circ} \tau_{\max }$. As the longest mode is determining for the viscosity, both expressions are identical from which $\tau_{\mathrm{d}}=2 / 5 \tau_{\max }$ results. This outcome, after substituting back $\tau_{\max }=5 / 2 \tau_{\mathrm{d}}$ into the BSW form is surprisingly or accidentally identical to the double reptation concept. This result is confirmed independently also from Figure 6.

The value of $M_{e}$ is estimated from the plateau modulus defined as $\mathrm{M}_{\mathrm{e}}=\rho \mathrm{RT} / \mathrm{G}_{\mathrm{N}}^{0}, 18$ where $\rho$ is the melt density and $R$ is the universal gas constant. $M_{e}$ is calculated to be $\sim 6200 \mathrm{~g} / \mathrm{mol}$ using the average value for $G_{N}^{0}$. The $Z$ value in this study extends up to $\sim 500$ and does not show different values for the plateau modulus. The estimated value of the entanglement molecular weight from the packing model with eq 11 a is $\sim 6800 \mathrm{~g} / \mathrm{mol}$ and agrees satisfactorily in view of the consistent approach in this work. Including the $4 / 5$ factor would yield $\mathrm{M}_{\mathrm{e}} \sim 4800$ or 5400 from the packing model.

Figure 3 (top) shows the derived experimental dependence of $\eta_{0}$ on $\mathrm{M}_{\mathrm{w}}$ at $\mathrm{T}=25{ }^{\circ} \mathrm{C}$. The open symbols correspond to earlier work of Gotro and Graessley ${ }^{5}$ and are included for comparison. Additionaly we have included inverse maximum frequencies of the normal mode process in similar polyisoprenes (right $\mathrm{Y}$-axis) as measured with dielectric spectroscopy, taken from Adachi et al. ${ }^{32,33}$ Theoretically the same molecular weight dependence as for the melt viscosity is to be found. They fit reasonably well to the data obtained in this investigation. To complete this comparison, the GraessleyGotro data $\left(\eta_{0} \sim \mathrm{M}^{3.6}\right)$ were also included in the data evaluation of our set since the microstructures seemed to be virtually identical. The fit corresponding to the joint-analysis of rheological data is shown as the thin dotted line in the lower part of Figure 3. The parameters of the $\log \eta_{0}$ vs $M$, i.e., $A=-14.54 \pm 0.47$ and $B=3.78$ \pm 0.10 , are only slightly different from the best values for the parameters in Figure 3 which correspond to our data only. To avoid systematic errors, however, the Gotro data are not used in the further data evaluations nor interpreted in this investigation. The scatter in both sets is very comparable (if our 100K sample is left out due to its additional discrepancy with SEC elution volumes in Figure 1) and only a relatively limited range in viscosity was covered. Instead, the range in this study spans the full unentangled regime $(Z<1)$ to the highly entangl ed regime with $Z \sim 500$. I n the high- $Z$ limit, the two available samples showed more experimental difficulties. One of them had a polydispersity index $\mathrm{M}_{\mathrm{w}} /$ $\mathrm{M}_{\mathrm{n}}=1.23$ and a first sign of this in the high-frequency slope of $G^{\prime \prime}(\omega)$ i.e., in the smaller $n_{e}$ slope. On the other hand, some extra deterioriation cannot be excluded upon mol ding and shaping the sample at the needed el evated temperatures in order to induce flow. Therefore, the narrower distributed sample was measured only in the

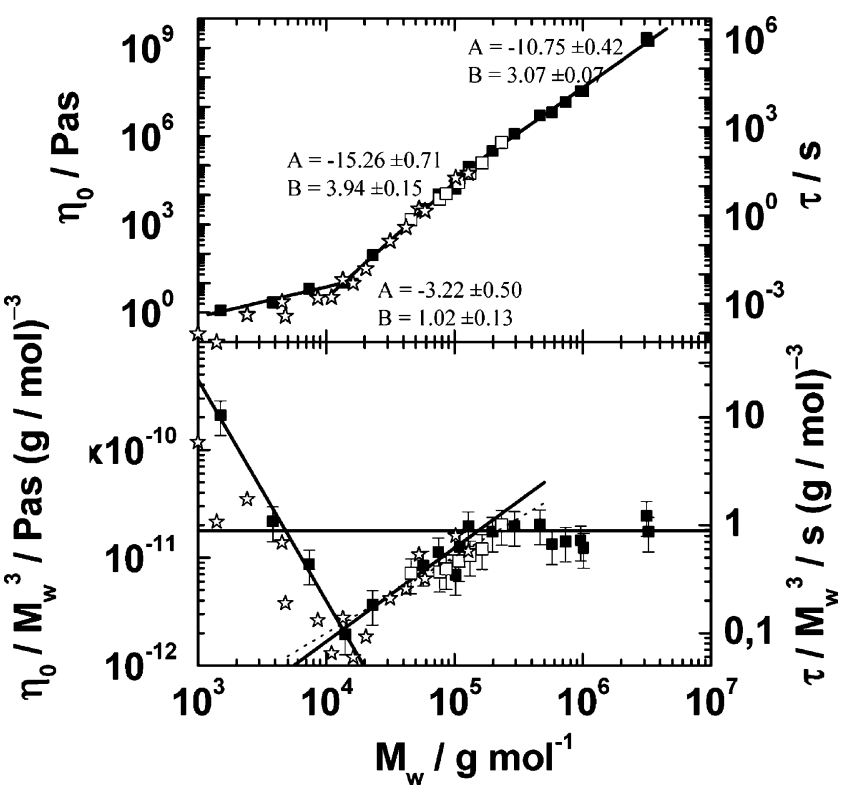

Figure 3. Top: Molecular weight dependence of zero shear rate viscosity for $\mathrm{PI}$ at $25^{\circ} \mathrm{C}$. $\eta_{0}$ is generally given as $\log \eta_{0}=$ $A+B \log M_{w}$. The parameters $A$ and $B$ for the different regimes are given in the text. Open symbols are the data from ref 5 . Inverse maximum frequencies $\tau$ (right axis) obtained from dielectic spectroscopy 32 shown as stars confirm the observed $M$ dependence. Bottom: $\eta_{0} / M_{w}{ }^{3}$ as well as $\tau / M_{w}{ }^{3}$ vs $M_{w}$. Solid lines correspond to the fitted equations (top) in the slope $=1,3.9$, and 3.0 region. The extrapolation from the slope $=3$ region to the Rouse region is included. Error bars were obtained assuming 5\% error on the respective molecular weight and the obtained errors in $\eta_{0}$. The $Y$-axis was obtained with the 3.07 exponent to include the full fitted equations consistently. The edges of the "magic" triangle represent $M_{e}^{*}$, $M_{c}$ and $M_{r}$ (see text). A joint analysis with the ref 5 data does not yield significantly different results and is discussed in the text. The fit of the joint analysis is represented by the thin dotted line in the lower part of the figure. Stars represent data taken from ref 32 and within reasonable error bars agree even at small $\mathrm{M}_{\mathrm{w}}$.

terminal region so as to avoid long-term exposure to high temperature during the measurement of the whole frequency range. Although polyisoprene degrades at higher temperatures as recently investigated by an in situ rheo-small-angle neutron scattering study 23 the melt viscosity (derived from the experimental peak maximum frequency) is still believed to be sufficiently accurate. Their values are given in parentheses in Table 2. The final results of this work are not affected, however, by the uncertainties in this sample or by some scatter at high frequency as the melt viscosities determined here differ only by $20-30 \pm 5 \%$ from the Graessley ones in the limited range.

The three different slopes with respective consecutive effective slopes 1.0, 3.9, and 3.0 from low $M_{w}$ to high $\mathrm{M}_{\mathrm{w}}$ are high-lighted in a reduced representation as shown in the lower part of $F$ igure 3 and discussed below. The $\eta \sim \mathrm{M}^{1}$ dependence is typical for the regime where entanglement effects are suppressed. Historically, the critical $M_{c}$ obtained at the crossing from $M^{1}$ to $M^{3.9}$ was taken as $2 \mathrm{M}_{\mathrm{e}}$. In this study, $\mathrm{M}_{\mathrm{c}}$ is experimentally determined from the graphical intersection to yield $M_{c}$ $=13300 \pm 1400 \mathrm{~g} / \mathrm{mol}{ }^{24}$ which is in good agreement with the 1964 values of 14000-15000 obtained by Fetters from concentrated solutions. ${ }^{25}$ The $M_{c}$ value of 13300 coincidentally leads to the ratio $\mathrm{M}_{d} / \mathrm{M}_{\mathrm{e}}=2.1 \pm$ 0.2 , whereas it is actually strongly packing-length dependent. Further, $\mathrm{M}_{\mathrm{c}}$ is in good agreement with the calculated value from the packing model as shown in 
Table 3 and follows the trend shown in eq $11 a-b$, where the $\mathrm{M}_{d} \mathrm{M}_{\mathrm{e}}$ ratio approaches 1 as $\mathrm{p}$ approaches $\sim 9.2 \AA$. The crossover to the pure reptation controlled regime here occurs at $\mathrm{M}_{r}$, i.e., the intersection $\mathrm{M}^{3.9}$ to $\mathrm{M}^{3}$. Data from ref 5 are shown as reference also in Figure 3 (open symbols).

From Figures 3 and 5 we observe following relationships:

$$
\begin{aligned}
\eta_{0} & =10^{-10.75 \pm 0.42} \mathrm{M}_{\mathrm{w}}^{3.07 \pm 0.07} \\
\eta_{0} & =10^{-15.26 \pm 0.71} \mathrm{M}_{\mathrm{w}}{ }^{3.94 \pm 0.15} \\
\eta_{0} & =10^{-3.22 \pm 0.50} \mathrm{M}_{\mathrm{w}}{ }^{1.02 \pm 0.13} \\
\tau_{\max } & =10^{-14.64 \pm 0.44} \mathrm{M}_{\mathrm{w}}{ }^{2.90 \pm 0.08} \\
\tau_{\max } & =10^{-18.82 \pm 1.10} \mathrm{M}_{\mathrm{w}}{ }^{3.68 \pm 0.36}
\end{aligned}
$$

A fair agreement with the slope for the relaxation time dependence 3.7 and viscosity is noticed. The experimental slope of $\mathrm{M}^{3.9}$ is slightly higher than earlier reported values of $3.6^{5}$ or $3.7^{14}$ for PI but still comparable. Values of 3.4-3.5 have been found for concentrated polyisoprene solutions. ${ }^{14,25,26}$ Dielectric spectroscopy measurements 32,33 on a very similar polyisoprene with slightly smaller vinyl content $(\sim 4 \%)$ also revealed a very comparable molecular weight dependence with a slope 4. The data from this study, shown also in Figure 3, add another independent confirmation of the derived $M_{c}$ value. The new slope of 3.0, however, is the theoretically predicted dependence of the tube model. I ts appearance is an additional proof of the tube concept. The transition from fluctuating-tube-controlled contributions to the melt viscosity to pure reptation (and more constraintrelease-controlled) dynamics occurs at the intersection 3.9 to 3 with $M_{r}=152700 \pm 24000 \mathrm{~g} / \mathrm{mol}$ using only the J ülich data. The corresponding crossover to reptational behavior using the full set of data (together with Gotro-Graessley) is located at approximately 218000 $\mathrm{g} / \mathrm{mol}$. This crossover event becomes accentuated if $\eta_{\mathrm{o}}$ $\mathrm{M}^{3}$ is plotted vs $\mathrm{M}$ as in Figure 3 . The relaxation time dependence on $M_{w}$ shows roughly the same behavior and from this, another independent estimate for $\mathrm{M}_{\mathrm{r}}$ from $\tau_{\max }$ Vs M (Figure 5) leads to approximately $\mathrm{M}_{\mathrm{r}} \sim 220000$ $\mathrm{g} / \mathrm{mol}$. The estimated value for $\mathrm{M}_{\mathrm{r}}$ on the basis of eq 11c, however, is $\sim 500000 \mathrm{~g} / \mathrm{mol}$ and therefore much higher and far outside the error bars. The difference is to be explained here: For the derivation of eq $11 c^{4}{ }^{4}$ the different behavior of melt viscosity $\eta_{0}$ on $\mathrm{M}_{\mathrm{w}}$ was used. If $\eta_{0}(\mathrm{M})$ is written in terms of $\eta_{0}\left(\mathrm{M}_{\mathrm{c}}\right)$ the ratio of both is $\left(\mathrm{M} / \mathrm{M}_{\mathrm{C}}\right)^{\alpha}$ at the lower $\mathrm{M}$-side and $\mathrm{QM}^{3} / \mathrm{M}_{\mathrm{e}}{ }^{2} \mathrm{M}_{\mathrm{C}}$ for pure reptation. The critical reptation molecular weight $M_{r}$ is defined where both meet. As constraint-rel ease has to do with the Rouse motion of the tube path itself, the prefactor Q above should bear CR effects as well. The published version of eq 11c was, however, strongly biased by (1) the neglect of the dependence on the viscosity exponent " $\alpha$ " in the contour-length-fluctuation controlled region (i.e., the slope or parameter $B$ in Figure 3 and for eq 11c taken always as 3.4), (2) the assumed numerical value for a constraint-rel ease corrected $Q$, and (3) the $M_{d} / M_{e}$ relationship. With all constants taken into account except CR, the front factor $\mathrm{Q}$ is determined as $15 / 4$ for the particular case that $\mathrm{M}_{\mathrm{c}}=2 \mathrm{M}_{\mathrm{e}}$. For $\mathrm{PI}$, by accident this is close to the case. Constraint-release processes additionally reduce the viscosity sensitively and only the prefactor Q should then be rescaled appropiately to a smaller value than
Table 3. Measured and Calculated PI Characteristic Molecular Weights at $25^{\circ} \mathrm{C}$

\begin{tabular}{cccc}
\hline $\begin{array}{c}\mathrm{M}_{\mathrm{e}}\left(10^{-3}\right. \\
\mathrm{g} / \mathrm{mol})\end{array}$ & $\begin{array}{c}\mathrm{V}_{\mathrm{e}}\left(10^{-3}\right. \\
\left.\mathrm{cm}^{3} / \mathrm{mol}\right)\end{array}$ & $\begin{array}{c}\mathrm{M}_{\mathrm{c}}\left(10^{-3}\right. \\
\mathrm{g} / \mathrm{mol})\end{array}$ & $\begin{array}{c}\mathrm{M}_{\mathrm{r}}\left(10^{-3}\right. \\
\mathrm{g} / \mathrm{mol})\end{array}$ \\
\hline $6.19( \pm 0.06)^{\mathrm{a}}$ & $11.4^{\mathrm{b}}$ & 13.1 & $220( \pm 50)$ \\
$6.74^{\mathrm{c}}$ & 12.4 & $13.7^{\mathrm{d}}$ & $437^{\mathrm{e}}$
\end{tabular}

a $\mathrm{M}_{\mathrm{e}}$ calculated from $\mathrm{G}_{\mathrm{N}}^{\circ}$. ${ }^{\mathrm{b}}$ Units of $\AA^{3}$. c Calculated from eq 11a. ${ }^{d}$ Calculated from eq 11b. e Calculated from eq 11c using fixed numerical constants.

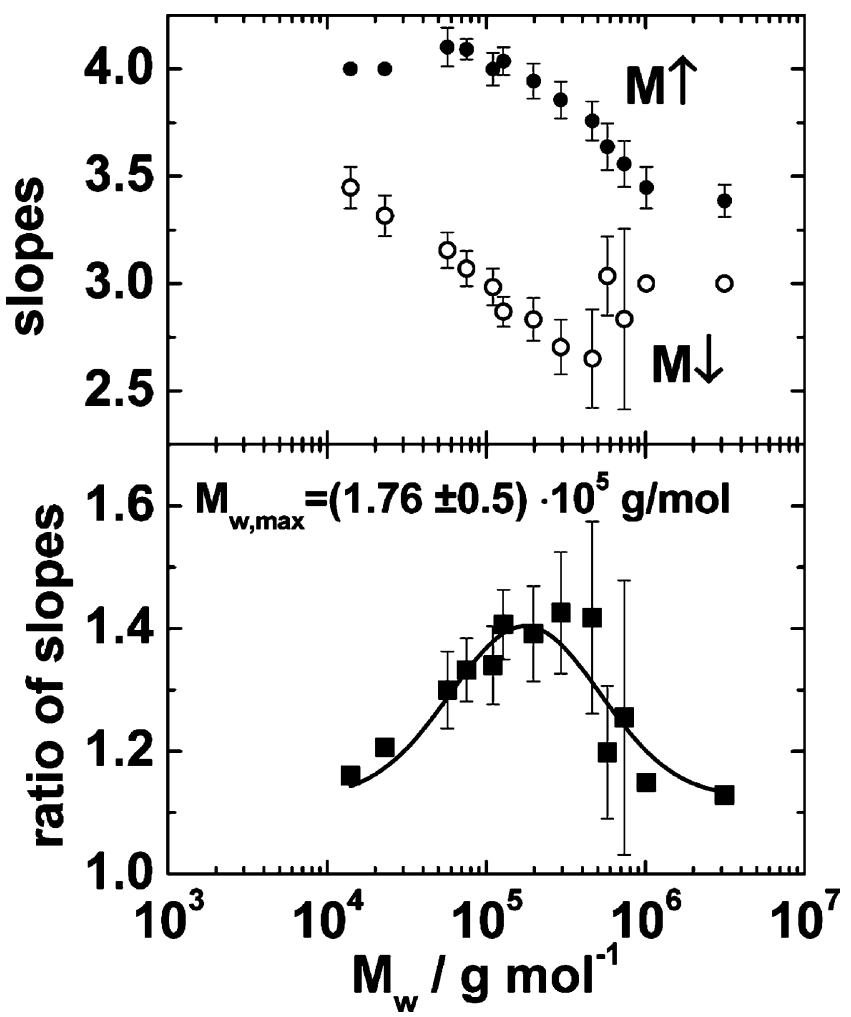

Figure 4. Accumulated mean slopes (see text) vs molecular weight (top). The lower part shows the ratio of (slope high-tolow) to (slope low-to-high). The solid line corresponds to a log Gaussian function fit. The maximum value of the fit function is included in the Figure. The data points without error bars were fixed to $4.0\left(\mathrm{M}^{\top}\right)$ and $3.0\left(\mathrm{M}_{\downarrow}\right)$, respectively.

$15 / 4=3.75$. The reduction to $30 \%$ of its value as was suggested earlier by Fetters et al. ${ }^{7}$ to give $\sim 1.13$ was physically wrong due to a confusing error in the used ref 4. Only the use of this particular wrong "Q" prefactor together with the fixed exponent 3.4 and fixed $M_{d} / M_{e}$ leads to the erroneous prediction for $M_{r}$. From the present study it should be possible to obtain an experimental estimate for $\mathrm{Q}$. Using the same arithmetic with a general exponent $\alpha$ for the CLF-controlled region in $\eta_{0}$, we end up with eq 16:

$$
\log M_{r}=\frac{\log \left(M_{c}^{1-\alpha} M_{e}^{2}\right)-\log Q}{3-\alpha}
$$

If no $C R$ contributions are observed, i.e., $Q=15 / 4$ and all formerly obtained critical molecular weights are plugged in, the crossover $M_{r}$ would be theoretically expected at $\sim 266000 \mathrm{~g} / \mathrm{mol}$. The amount of actual constraint-release can be estimated from the experimental crossover reptational $\mathrm{M}_{\mathrm{r}}$. With eq 16 and the span in $\mathrm{M}_{\mathrm{r}}$ from the considerations before, we derive the prefactor " $Q$ " to about $2-3$ i.e., $\sim 2 / 3$ of $15 / 4$. For the Graessley model which described CR in terms of a number $\mathrm{x}$ of sideways tube hops ${ }^{16}$ and a consequent 
reduction of the terminal time by $(\pi / 12)^{x} / x$, this would correspond to about $\mathrm{x}=1.5$ or 2 hops on average per chain. The former misinterpretation in which the factor $(1 / x)$ was missing, however, may affect the full extrapolation of the packing model in ref 7 to large packing lengths since the respective viscosity dependence must be known for all $\mathrm{p}$. This is to be explored in forthcoming work. In addition, the sensitivity of $\mathrm{M}_{\mathrm{r}}$ is about $100 \%$ if the $\alpha$-exponent varies only by 0.2 . As the crossing into this regime is, however, experimentally clear from our data (i.e. roughly at about $Z \sim 30$ ), it is evident that a more sophisticated description of the packing model at this point and better theoretical corrections are necessary. We conclude here that experimentally CLF (I ower $M$ ) as well as CR (higher $M$ ) are acting simultaneously at the onset of reptation which seems to appear between 152700 and $220000 \mathrm{~g} / \mathrm{mol}$ roughly for PI.

The onset of $M_{r}$ can be probed via an optimized version of a procedure earlier introduced by $\mathrm{Colby}^{29}$ (Figure 4). From high $\mathrm{M}_{\mathrm{w}}$ to lower $\mathrm{M}_{\mathrm{w}}$ 's the accumulated mean slope in $M$ should drift away from 3.0 if mixed with the steeper slope at lower M. At the same time, in the reverse way, the slope should decrease if mixed with the shallower slope of 3 at high $\mathrm{M}_{\mathrm{w}}$. The crossover molecular weight, i.e., $\mathrm{M}_{\mathrm{r}}$ by definition, is then accentuated by dividing the effective slopes as shown in Figure 4. A fitted (arbitrary) log-Gaussian function is symmetrical around $M_{r} \sim 175000 \pm 50000 \mathrm{~g} / \mathrm{mol}$. The vertical axis $A / B$ in Figure 4 is the ratio of (slope highto-low) to (slope low-to-high) at the corresponding molecular weight ( 3.9/2.9 in the maximum). The measured $M_{r}$ is thus not influenced by the assumption that the transition between the two slopes is sharp and discrete, nor is it determined by a wrong assumption of the slope in the tube-controlled region or assumptions for $M_{c}$. The critical number of entanglements, $Z_{c r i t}$, at which this occurs is again situated at $Z \sim 28 \pm 8$ with $\mathrm{M}_{\mathrm{e}}=6200$. This is much less than considered in ref 19 and somewhat higher than recently found from self-and tracer diffusion coefficients ${ }^{23}$ and melt viscosity. ${ }^{34}$ This approach allows the selection of data points to both directions around $\mathrm{M}_{\mathrm{r}}$ and as such indirectly sustains the $\mathrm{M}_{\mathrm{C}}$ determination. It is to be investigated in forthcoming reevaluations of $\mathrm{PIB}, \mathrm{PB}, \mathrm{PE}$, and $\mathrm{PS}$ data ${ }^{30}$ whether the pure reptation limit for $Z$ al ways occurs at the same $\mathrm{M} / \mathrm{Me}_{\mathrm{e}}$

As $M_{w}$ increases the relaxation time also shifts considerably to longer times or low frequencies as $\tau_{\mathrm{d}} \sim$ $Z^{3} \tau_{\text {e. }}$. It proved impossible as stated before to measure the full flow regime in shear for the highest $M_{w}$ sample and a creep experiment would have been more appropriate. Instead, we have used a different approach. As the peak position of the measured loss peak is by definition $\tau_{\mathrm{d}}{ }^{-1}$, which can be located easily from linlog representations of data and which is related as before to the longest relaxation time $\tau_{\max }$, a linear empirical relation between $\tau_{\mathrm{d}}$ and $\eta_{0}$ can be set up as in Figure 6 . The melt viscosity of an unmeasurable substance due to the limited frequency window could then be estimated to good accuracy without having access to the full flow regime. This approximation was used for the $M_{w}=3 \times 10^{6}$ sample and verified by the full range of available molecular weights for which the peaks were identified uniquely. A relationship $\eta_{0}=10^{5.39} \tau_{\mathrm{d}}{ }^{1.01}$ for $\mathrm{PI}\left(25^{\circ} \mathrm{C}\right)$ is derived. This agrees with the experimental relationships from the $\mathrm{H}$-spectrum-based fits in eq 15 if $\tau_{\max }$ (corrected to $\tau_{\mathrm{d}}$ ) and $\eta_{0}$ with $\mathrm{M}^{3}$ and $\mathrm{M}^{3.9}$ (Figures 3 and 5) are divided by each other to eliminate the $\mathrm{M}_{\mathrm{w}}$ dependence, yielding $\eta_{0}=10^{4.28} \mathrm{M}^{0.17} \tau_{\mathrm{d}}^{1.0}$. The remain-

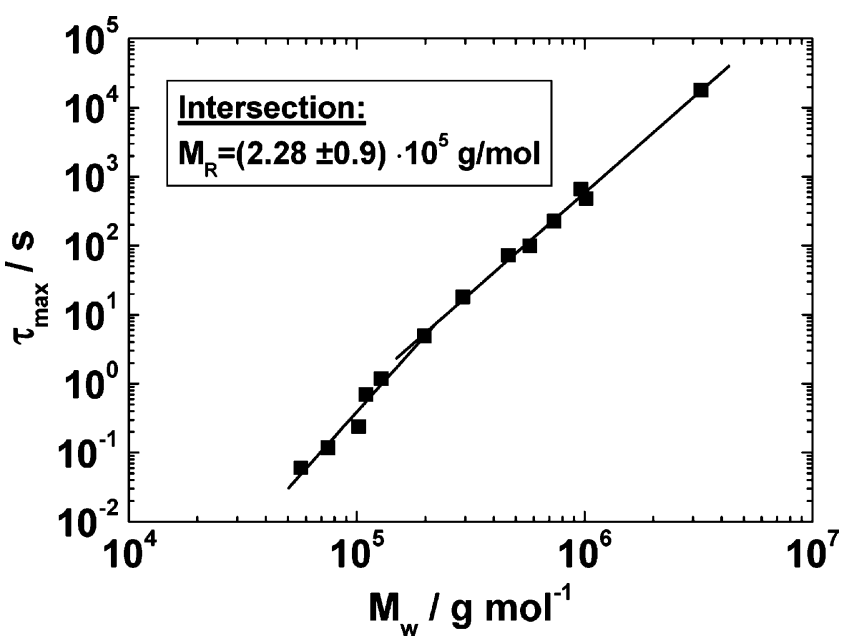

Figure 5. Molecular weight dependence of longest BSW relaxation time for $\mathrm{PI}$ at $25^{\circ} \mathrm{C}$. Same convention as in Figure 3 for $A$ and $B$, values given in the text.

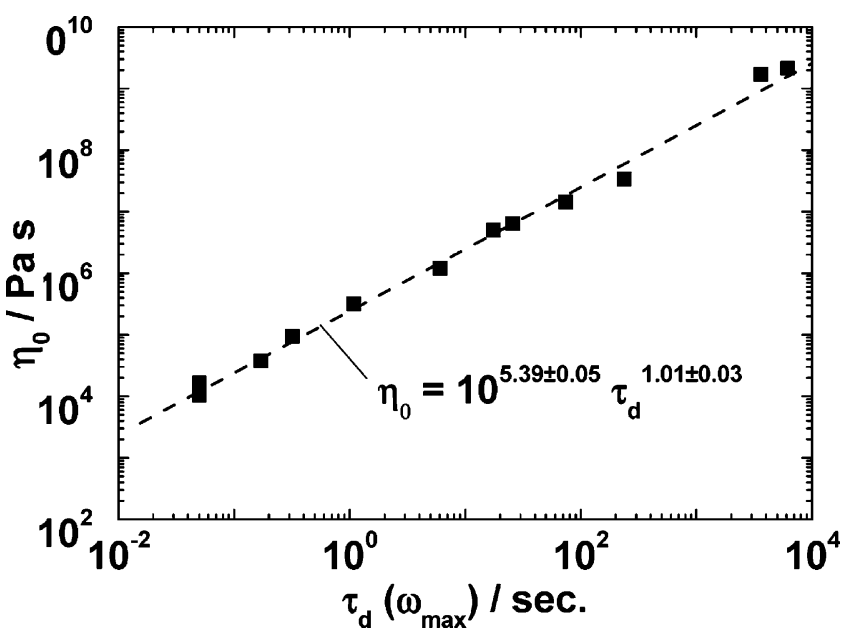

Figure 6. Fitted zero-shear viscosity vs the reptation time obtained from the maximum in the loss peak. The dashed line is the best fit and the prefactor $\left(10^{5.39}\right)$ corresponds to the expected value basing on $\mathrm{G}_{\mathrm{N}}{ }^{0}$ and $\mathrm{n}_{\mathrm{e}}$.

ing correction $\mathrm{M}^{0.17}$ is due to the small differences in the exponents. For the spanned molecular weight range, this $\mathrm{M}$-correction is situated between $10^{0.85}$ and $10^{1}$ or at $\mathrm{M}_{\mathrm{r}}$ about $10^{0.93}$ and so leads to $\eta_{0}=10^{5.28} \tau_{\mathrm{d}}$. $\tau_{\mathrm{d}}$ from the peak position and BSW characterization agree well. In view of the log scale, the discrepancy is negligibly small and can be easily absorbed in small uncertainties in $\mathrm{M}_{\mathrm{w}}$. The excell ent agreement of both reptation times, however, corroborates the inherent assumption in the BSW-molecular rheology transciption, regarding the $\tau_{\text {max }} / \tau_{\mathrm{d}}$ ratio as provided earlier to be simply related to the $\mathrm{n}_{\mathrm{e}}$ exponent. Table 2 yiel ds a ratio of $\tau_{\max } / \tau_{\mathrm{d}}\left(\omega_{\max }\right)$ of $3.1 \pm 0.9$, which is nearly 2.5. A calculation of the C-factor as in eq 14 basing on $\tau_{\mathrm{e}}$ from BSW fits with Z, combined with the observed peak maximum $\tau_{\mathrm{d}}$ with $\mathrm{C}=\sqrt{\mathrm{Z}}\left(1-\sqrt{\tau_{\mathrm{d}} / 3 \mathrm{Z}^{3} \tau_{\mathrm{e}}}\right)$ yields $\mathrm{C} \sim 1.83 \pm 0.15$, only marginally different from the value of 1.47 used.

This reinvestigation of model 1,4-polyisoprenes has linked all possible regimes with a consistent description of the dynamic moduli in seemingly well-parametrized mol ecular-rheological parameters. It is thus possible to obtain an estimate for the monomeric friction coefficient $\xi_{0}$ at $25{ }^{\circ} \mathrm{C}$. To date, the value used has been the estimate Hevea rubber listed in Ferry ${ }^{18}$ as $3.89 \times 10^{-10}$ $\mathrm{N} \mathrm{s} / \mathrm{m}$ and obtained from a lightly cross-linked material. 
Several expressions are available from which the Rouse theory yields unapproximated

$$
\xi_{0}=\frac{36 \mathrm{M}_{0}^{2} \eta_{0}}{\rho \mathrm{a}^{2} \mathrm{~N}_{0} \mathrm{M}}
$$

Here, $M_{0}$ is the monomer mass and $a$ is the effective statistical segment length. In the unentangl ed regime, the associated slightly higher power $\sim 1.1$ (compared to 1) does not affect the interpretation. Chain end effects have been eliminated by shifting the reference temperature. With the estimate for $\eta_{0} / \mathrm{M}$, the mean monomeric friction coefficient $\xi_{0}$ is $3.27 \times 10^{-10} \mathrm{~N} \mathrm{~s} / \mathrm{m}$. This compares very well indeed to the Hevea rubber estimate. ${ }^{18}$ If one considers the maximum limits, given by the error bars on $\eta_{0} / \mathrm{M}$, the full range of (1.03-10.3) $\times$ $10^{-10} \mathrm{~N} \mathrm{~s} / \mathrm{m}$ is acceptable. The NSE estimate is about 3 times higher 22 than the former mean value of $3.27 \times$ $10^{-10} \mathrm{~N} \mathrm{~s} / \mathrm{m}$ but is inside the interval. This higher estimate was determined on a well-entangled system in the Rouse-time regime and probably slightly different WLF parameters are responsible for this difference.

Since now all power-laws for the melt viscosity are known, a final test of the Doi-Edwards tube concept predictions can be made. For this, we refer again to the lower part of Figure 3. In this context, the intersection of low with high $M_{w}$ dependence in Figure 3 can be thought of as that basic molecular weight, $\mathrm{Me}_{\mathrm{e}}^{*}$ which should be devoid of contour length fluctuation contributions if the contour-length-fluctuation mechanism were the single cause of the 3.4-3.9 power. This is an inherent property due to the assumptions of the tube model itself. Using the average value of the prefactor from the pl ot yields an $\mathrm{Me}^{*}$ of $4700 \pm 500 \mathrm{~g} / \mathrm{mol}$. Figure 3 , which shows this extrapolation very decoratively, therefore characterizes a "triangle" with edges $\mathrm{M}_{\mathrm{e}}{ }^{*}, \mathrm{M}_{\mathrm{C}}$, and $\mathrm{M}_{\mathrm{r}}$. The triangle therefore is a measure for the contribution of CLF and CR. The entanglement mass $\mathrm{M}_{\mathrm{e}}{ }^{*}$ differs only slighly from the value obtained from the plateau modulus itself and we may identify both here first for simplicity. It agrees, however, with the value if the factor $4 / 5$ is included in the plateau modulus calculation. This question is currently under investigation. ${ }^{30}$ It is to be expected that this method is likely to work for the determination of $\mathrm{M}_{\mathrm{r}}$ for other polymers in the reverse way, e.g., if contour length fluctuations are dominant or when long chains cannot be synthesized for chemical reasons. A decent estimate for $M_{r}$ can then still be obtained even from a type of pl ot as Figure 3 or 4 if only a limited portion of the fluctuation-controlled viscosity range law is known together with the plateau modulus. It reraises the question whether there is a molecular origin of the critical molecular weight for the tube-onset as the reptation mechanism extrapolates between both limits. One reason could be that unentangl ed chains al ways look smaller (due to free ends, contour-length fluctuations, and even deviations from Gaussian statistics) and therefore need a seemingly higher mass to accommodate the measured viscosity. The plateau modulus, or equivalently $M_{e}$, concerns primarily the high frequency limit of the storage modulus and is less or not sensitive to chain length effects. Clearly the melt viscosity depends on the actual endto-end distance and long times. The resulting correlation and combination of $M_{e}$ with $M_{r}$ as shown here is therefore natural and should be given more priority than relations to $M_{c}$. Structural and final experiments are still lacking at this point whereas recent NSE investigations have confirmed the CLF process. ${ }^{28}$
Table 3 summarizes the polyisoprene rheological mol ecular weights determined in this study. The error bar on both $M_{c}$ and $M_{r}$ are obtained from error propagation. The error bar on $\mathrm{M}_{\mathrm{e}}$ is taken from the Winter-fit of the plateau modulus.

\section{Conclusion}

In this work we have reinvestigated the rheological properties of a series of PI samples having molecular weights in the range $1500-3200000 \mathrm{~g} / \mathrm{mol}$. The value of the plateau modulus obtained was $0.37 \mathrm{MPa}$ in good agreement with the literature value of 0.35 . The relation between the melt viscosity, $\eta_{0}$ and the weight-average mol ecular weight, $M_{w}$ is evaluated over this wide range of the molecular weight. The characteristic molecular weights are redetermined $\left(\mathrm{M}_{\mathrm{e}}, \mathrm{M}_{\mathrm{c}}\right.$, and $\left.\mathrm{M}_{\mathrm{r}}\right)$ and compared to their estimates from packing model parameters. A new "triangl e" was suggested which could ease the prediction of one unknown characteristic mol ecular weights if slopes are known. The monomeric fraction coefficient $\xi_{0}$ was determined in this study for the first time for anionically polymerized PI. The longest empirical relaxation time $\tau_{\max }$ was related to the reptation time, $\tau_{\mathrm{d}}$ and calculated in different ways as is also the case for $\tau_{\mathrm{e}}$. From the peak maximum in G" $(\omega), \omega_{\max }$ as $\tau_{\mathrm{d}}=1 / \omega_{\max }$ and the measured viscosities, a linear equation which allowed extrapolation to the high $\mathrm{M}_{\mathrm{w}}$ limit was developed. We have experimentally shown that $\tau_{\mathrm{d}}$ or $\tau_{\max }$ vs $\mathrm{M}_{\mathrm{w}}$ had the same trend as $\eta_{0}$ vs $\mathrm{M}_{\mathrm{w}}$ over the full interval of molecular weights. The experimental data were analyzed consistently using the BSWmodel using the empirical relaxation spectrum and excellent fits were obtained. BSW parameters for all PI samples could be determined and a correlation with microscopic parameters from molecular-rheol ogical models was tested. The model could provide a quick alternative to molecularly based approaches. The transition to reptation is found to happen between $Z_{c} \sim 25-35$. It is concluded that the predictions for the reptational molecular weight must be reanalyzed carefully for different polymers. Indirect proof for CLF and CR is observed from multiple ways to fix latter crossover molecular mass.

Acknowledgment. Support for this research was provided by the International Bureau, J ülich, Germany. Helpful discussions with Ekkehard Straube, University of Halle, Halle, Germany, and Alexei Likhtman of IRC, Leeds, U.K., are gratefully acknowledged as is the hel $p$ by Marlies Hintzen, FZJ -IFF for the SEC and LS polymer characterization. Likewise, Willy Bünten, FZJ IFF, is thanked for his help with some rheological recharacterization of the samples.

\section{References and Notes}

(1) Benallal, A.; Marin, G.; Montfort, J .; Derail, C. Macromolecules 1993, 26, 7229.

(2) Baumgaertel, M.; Schausberger, A.; Winter, H. H. Rheo. Acta 1990, 29, 400 .

(3) Baumgaertel, M.; De Rosa, M. E.; Machado, J .; Masse, M.; Winter, H. H. Rheo Acta 1992, 31, 75.

(4) Graessley, W. W. J . Polym. Sci., Polym. Phys. Ed. 1980, 18, 27.

(5) Gotro, J .; Graessley, W. W. Macromolecules 1984, 17, 2767.

(6) Fetters, L.J .; Lohse, D. J .; Richter, D.; Witten, T. A.; Zirkel, A. Macromolecules 1994, 27, 4639.

(7) Fetters, L. J .; Lohse, D. J .; Milner, S. T.; Graessley, W. W. Macromol ecules 1999, 32, 6847.

(8) Doi, M. J . Polym. Sci., Polym. Lett. Ed. 1981, 19, 265

(9) Doi, M.; Edwards, S. F. The Theory of Polymer Dynamics; Clarendon Press: Oxford, U.K., 1986. 
(10) Larson, R. G.; TheStructureand Rheol ogy of Complex Fluids, Oxford University Press: New York, and Oxford, U.K., 1999; p 74.

(11) F etters, L. J .; Lohse, D. J .; Graessley, W. W. J . Polym. Sci., Part B: Polym. Phys. 1999, 37, 1023.

(12) Fetters, L. J .; Lohse, D. J .; Colby, R. H. Physical Properties of Polymers Handbook; Mark, J . E., Ed.; AIP Press: Woodbury, NY, 1996; p 335.

(13) J ackson, J.; De Rosa, M. E.; Winter, H. H. Macromolecules 1994, 27, 2426.

(14) Graessley, W. W.; Masuda, T.; Roovers, J .; Hadjichristidis, N. Macromol ecules 1976, 9, 127.

(15) Floudas, G.; Gravalides, C.; Reisinger, T.; Wegner, G. J . Chem. Phys. 1999, 111, 9847.

(16) Roovers, J .; Toporowski, P. M. Rubber Chem. Technol. 1990, 63, 734.

(17) Floudas, G.; Meramveleotaki, K.; Hadijichristidis, N. Macromolecules 1999, 32, 7496.

(18) Ferry, J . D. Viscoelastic Properties of Polymers, 3rd ed.; Wiley, New York, 1980.

(19) Likhtman, A.; McLeish, T. C. B. Macromolecules 2002, 35, 6332.

(20) Milner, S. T.; McLeish, T. C. B. Phys. Rev. Lett. 1998, 81 , 725.

(21) Wang, S. J . Polym. Sci., Polym. Phys. 2003, 41, 1589.

(22) Richter, D.; Butera, R.; Fetters, L. J .; Huang, J. S.; Farago, B.; Ewen, B. Macromolecules, 1992, 25, 6156.
(23) Botti, A.; Pyckhout-Hintzen, W.; Richter, D.; Straube, E. Rheol. Acta 2002, 41, 475.

(24) Nemoto, N.; Odani, H.; Kurata, M. Macromol ecules 1972, 5, 531.

(25) Fetters, L. J .J . Res. Natl. Bur. Stand. U.S.A. 1965, 69A, 33.

(26) Hadjichristidis, N.; Roovers, J . J . Polym. Sci., Polym. Phys. Ed. 1974, 12, 2521.

(27) Fetters, L. J .; Graessley, W. W.; Kiss, A. D. Macromolecules 1991, 24, 3136.

(28) Wischnewski, A.; Monkenbusch, M.; Willner, L.; Richter, D.; Likhtman, A.; McLeish, T. C. B.; Farago, B. Phys. Rev. Lett. 2002, 88, 058302.

(29) Colby, R. H.; Fetters, L. J .; Graessley, W. W. Macromolecules 1987, 20, 2226.

(30) Kahle, S.; Pyckhout-Hintzen, W.; Goad, M.; Richter, D.; Fetters, L. J . Manuscript in preparation, 2004.

(31) Garcia-Leiner, M.; Manero, O.; Herrera, R. Rheol. Acta 2003, 42, 171.

(32) Imanishi, Y.; Adachi, K.; Kotaka, T. J . Chem. Phys. 1988, 89, 7585.

(33) Adachi, K.; Kotaka, T. J . Macromolecules 1985, 18, 466.

(34) Vega, J .; Rastogi, S.; Peters, G.; Meijer, H. J . Rheol. 2004, $48,663$.

MA030557+ 